\title{
Correction to: Pharmacologically induced impairment of neurovascular coupling responses alters gait coordination in mice
}

\author{
Stefano Tarantini • Andriy Yabluchanskiy • Gábor A. Fülöp • Peter Hertelendy • M. Noa \\ Valcarcel-Ares • Tamas Kiss • Jonathan M. Bagwell • Daniel O'Connor • Eszter Farkas • \\ Farzaneh Sorond • Anna Csiszar • Zoltan Ungvari
}

Published online: 21 February 2018

(C) American Aging Association 2018

Correction to: GeroScience (2017) 39:601-614. https://doi.org/10.1007//11357-017-0003-x

The original version of this article unfortunately contained an error.

Andriy Yabluchanskiy's name was incorrectly spelled as Andriy Yabluchanksiy in the original version. The correct spelling is now reflected in this article.

The online version of the original article can be found at https://doi.org/10.1007/s11357-017-0003-x

S. Tarantini · A. Yabluchanskiy · G. A. Fülöp •

P. Hertelendy • M. Noa Valcarcel-Ares • T. Kiss •

J. M. Bagwell · D. O’Connor · A. Csiszar · Z. Ungvari $(\bowtie)$

Reynolds Oklahoma Center on Aging,

Department of Geriatric Medicine,

University of Oklahoma Health Sciences Center,

Oklahoma City, OK, USA

e-mail: zoltan-ungvari@ouhsc.edu

S. Tarantini · A. Yabluchanskiy · G. A. Fülöp ·

P. Hertelendy $\cdot$ A. Csiszar $\cdot$ Z. Ungvari

Translational Geroscience Laboratory,

Department of Geriatric Medicine, University of

Oklahoma Health Sciences Center,

Oklahoma City, OK, USA

\author{
G. A. Fülöp \\ Division of Clinical Physiology, Faculty of Medicine, University \\ of Debrecen, Debrecen, Hungary \\ J. M. Bagwell \\ Department of Veterinary Pathobiology, Oklahoma State \\ University, Stillwater, OK, USA \\ E. Farkas · A. Csiszar · Z. Ungvari \\ Department of Medical Physics and Informatics, Faculty of \\ Medicine and Faculty of Science and Informatics, University of \\ Szeged, Szeged, Hungary \\ F. Sorond \\ Department of Neurology, Northwestern University, Chicago, IL, \\ USA
}

